\title{
An audit of operating theatre utilisation and day-of-surgery cancellations at a regional hospital in the Durban metropole
}

\author{
I I Asmal, MB ChB, DA (SA); K Keerath, MB BCh, DA (SA), FCA (SA), MMed (Anaesth); L Cronjé, MB ChB, FCA (SA) \\ Discipline of Anaesthesiology and Critical Care, Nelson R Mandela School of Medicine, College of Health Sciences, University of KwaZulu-Natal, \\ Durban, South Africa
}

Corresponding author: I I Asmal (asmimr001@myuct.ac.za)

Background. Operating theatres account for a significant proportion of hospital costs. There is a paucity of data evaluating utilisation of South African (SA) state operating theatres.

Objectives. To measure operating theatre utilisation and the rate of day-of-surgery cancellations (DOSCs) in a state hospital theatre complex. Methods. A prospective audit of a state operating theatre complex at a Durban regional hospital was performed between 26 February and 26 April 2018. Times were collected for each theatre case from the entry of the patient into theatre to their departure to the post-anaesthetic care unit. This was done on weekdays between $08 \mathrm{~h} 00$ and $16 \mathrm{~h} 00$. The factors causing any delays and DOSCs were identified and recorded. Results. Over the study period, 125220 operative minutes were available for both elective and emergency operating theatres; 655 elective cases and 359 emergency cases were performed. Overall theatre utilisation was $55.2 \%$, with actual operating time comprising only $36.9 \%$ of all available time. Non-operative time occupied $63.1 \%$ of all available time, split between late starts (9.3\%), early list finishes (16.1\%), changeover times (19.4\%) and anaesthetic time (18.3\%). The DOSC rate was $26.2 \%$, with 232 cases cancelled on the day of surgery. Just under half of the DOSCs were avoidable. The most common reason for cancellation was lack of operative time.

Conclusions. Measured theatre utilisation was higher than previously quoted figures for SA state hospitals, but below international benchmarks. A significant amount of time was wasted as a result of delayed first-case starts, prolonged changeovers and early terminations of lists, all of which contributed to a high DOSC rate. Before more theatre time can be made available, theatre users must first optimise use of currently available time. Further studies quantifying the effect of staff shortages in state operating theatres on inefficient use of time are required.

S Afr Med J 2019;109(10):765-770. https://doi.org/10.7196/SAMJ.2019.v109i10.13815

Operating theatres account for a significant proportion of hospital costs. ${ }^{[1]}$ Escalating healthcare costs and limited state health budgets necessitate an efficient theatre service to maximise service delivery and reduce costs. ${ }^{[2]}$ The Lancet Commission has highlighted the need for scaling up surgical services in low- and middle-income countries to increase access to essential surgery. ${ }^{[3]}$ Scale-up will place an increased burden on existing resources, further emphasising the importance of optimising use of available theatre time. ${ }^{[2]}$ International studies have shown that $16-24 \%$ of operating time may be wasted as a result of inefficient running of the operating theatre complex. ${ }^{[4,5]}$ A study from a South African (SA) private hospital group showed overall theatre utilisation of $48 \%$, well under the benchmark of 70 $80 \%{ }^{[6]}$ but there is a paucity of data from state hospitals. ${ }^{[2]}$

\section{Objectives}

To determine whether a state hospital's operating theatre time was used efficiently and to identify areas where improvements could be made. We also sought to determine the rate of day-of-surgery cancellations (DOSCs) and to identify whether these could be avoided.

\footnotetext{
Methods

Study design and setting

We conducted a prospective audit evaluating time utilisation and DOSCs in an operating theatre complex at a Durban regional hospital from 26 February to 26 April 2018. Ethics approval was obtained from the University of KwaZulu-Natal Biomedical Research Ethics Committee (ref. no. BE016/18) and the KwaZulu-Natal Department of Health (ref. no. KZ_201802_007). All weekday elective and
}

emergency lists between $08 \mathrm{~h} 00$ and $16 \mathrm{~h} 00$ were included. Lists during these times over public holidays or weekends were excluded.

\section{Data collection, outcome variables and definitions}

A data collection tool was designed and completed by the attending anaesthetist. Five different times were recorded (Table 1) and reasons for delays were documented. To improve the accuracy of data collection, all theatre users were informed of the study, trained on use of the data collection tool and encouraged to report delays. Where times were missing, they were completed by using the operating theatre record available in the theatre complex. Outcome definitions are provided in Table 1. Outcome variables were extrapolated from the data collection tool. DOSCs and reasons for cancellation were recorded separately at the end of each theatre day.

- Late first-case starts were defined as a start time delay of $>15$ minutes from the scheduled start time for the operating theatre list. For emergency theatres, late first-case starts were taken as the total unused time prior to the first emergency case commencing, when no activity occurred in the emergency theatre.

- Prolonged changeover time was defined as turnover times $>60$ minutes. ${ }^{[7]}$

- Anaesthetic time was calculated as the time from arrival of a patient in the operating room to skin incision and the time following wound closure to exiting from the operating room. It included patient transfer to the operating bed, induction and positioning, cleaning and draping, as well as reversal of anaesthesia and transfer out of theatre.

- Theatre utilisation was calculated as the sum of the anaesthesia and surgical times over total available time, excluding early 
starts and over-running hours. Changeover time, while deemed essential, is 'non-value adding' and was therefore excluded from the calculation of theatre utilisation. ${ }^{[8]}$

- The DOSC rate was calculated as the number of elective cases cancelled on the day of surgery over the total number of scheduled elective cases. DOSCs and intraoperative and slate delays were then analysed to determine whether they were avoidable or nonavoidable. 'Avoidable' was defined as a cancellation or delay that occurred as a result of situations that existed prior to the day of surgery and could have been avoided by careful review and communication between patients and/or staff. ${ }^{[9]}$ 'Unavoidable' was defined as a delay or cancellation that could not have been avoided even with adequate review or communication between patients and/or staff. ${ }^{[9]}$

\section{Statistical analysis}

It was determined that the minimum required sample recruitment time was 40 days for all variables, which would enable us to obtain a sample of $\sim 1000$ operating theatre cases based on knowledge of the theatre complex's past caseloads and allow us to detect any significant difference from international benchmarks with a probability of $95 \%$. Data were captured on a data analysis spreadsheet, using Excel 2013 (Microsoft, USA). Analysis was performed using SPSS statistics version 23.0 (IBM, USA). Timebased variables (minutes) for each theatre were reported as means, standard deviations (SDs) and ranges, and counts and percentages were used for categorical data.

\section{Results}

The study was conducted on five elective and two emergency operating theatres, across the general surgery, orthopaedic, and obstetrics and gynaecology disciplines. We evaluated 266 theatre lists, consisting of 184 elective lists and 82 shared emergency lists. A total of 1014 cases comprised 655 elective cases and 359 emergency cases. There was a total of 125220 available operative minutes. Table 2 displays the distribution of cases, lists and utilisation of individual theatres

Table 1. Times documented and extrapolated per theatre case and session

\begin{tabular}{ll}
\hline Times documented & Definition \\
\hline Time A & Time patient enters theatre \\
Time B & Anaesthesia start time \\
Time C & Operative start time \\
Time D & Operative end time \\
Time E & Time patient leaves the operating theatre \\
Times extrapolated & Outcome definition \\
\hline Total available time & All time within the allocated theatre sessions excluding over-running times and early start times. \\
Late start time & Time A - scheduled start time \\
Anaesthesia time & (Time C - Time A) + (Time E - Time D) \\
Changeover time & Time A (next patient) - Time E (previous patient) \\
Prolonged changeover & Changeover time $>60$ minutes \\
Surgical time & Time D - Time C \\
Over-running time & Surgical time and anaesthesia time occurring after the scheduled end time of the operating theatre list \\
Early terminations & Scheduled end time of list - Time E (last patient) \\
&
\end{tabular}

Table 2. Overall time utilisation in the theatre complex and utilisation by discipline

\begin{tabular}{|c|c|c|c|c|c|}
\hline \multirow[b]{2}{*}{ Discipline } & \multicolumn{5}{|c|}{ Time utilisation in the operating rooms } \\
\hline & & \multicolumn{2}{|l|}{ Cases done, $n$} & Lists allocated, $n$ & Utilisation, \% \\
\hline \multicolumn{2}{|c|}{ Obstetrics and Gynaecology (Emergency) } & 165 & & 41 & 53.3 \\
\hline \multicolumn{2}{|l|}{ Obstetrics 1} & 142 & & 36 & 42.5 \\
\hline \multicolumn{2}{|c|}{ Obstetrics and Gynaecology (Elective) } & 177 & & 40 & 56.7 \\
\hline \multicolumn{2}{|l|}{ General (Emergency) } & 194 & & 41 & 52.9 \\
\hline \multicolumn{2}{|l|}{ General Surgery } & 133 & & 37 & 57.2 \\
\hline \multicolumn{2}{|l|}{ Orthopaedic Surgery 1} & 116 & & 40 & 81.2 \\
\hline \multicolumn{2}{|l|}{ Orthopaedic Surgery 2} & 87 & & 31 & 61.9 \\
\hline \multirow[t]{2}{*}{ Overall } & & \multicolumn{2}{|l|}{1014} & 266 & 55.2 \\
\hline & \multicolumn{5}{|c|}{ Overall time utilisation in the theatre complex } \\
\hline Activity & Total time (min) & Mean (SD) (min) & 95\% CI (min) & Range (min) & $\begin{array}{l}\% \text { of total } \\
\text { available time }\end{array}$ \\
\hline Time lost due to late start & 11640 & $44(39)$ & $39-49$ & $5-210$ & 9.3 \\
\hline Time lost due to early finish & 20100 & $76(70)$ & $68-84$ & $4-350$ & 16.1 \\
\hline Total changeover time & 24300 & $24(20)$ & $22-26$ & $0-253$ & 19.4 \\
\hline Total anaesthesia time & 22920 & $23(19)$ & $21-24$ & $7-77$ & 18.3 \\
\hline Total surgical time & 46260 & $46(38)$ & $43-48$ & $2-305$ & 36.9 \\
\hline Total time available & 125220 & - & - & - & 100 \\
\hline
\end{tabular}


and the overall breakdown of different time utilisations of the theatre complex.

\section{Late first-case starts}

Total time lost due to late first-case starts was 11640 minutes, $9 \%$ of total available time. The mean (SD) first-case start delay for the theatre complex was 44 (39) minutes. The majority of elective first-case starts were delayed (136/184, $74 \%)$. The mean (SD) first-case start delay was 47 (31) minutes for elective lists. Only 25/184 elective lists (14\%) commenced on time, while 23/184 elective lists (13\%) commenced early. Emergency theatres were unoccupied for a mean (SD) of 71 (42) minutes prior to the first emergency case commencing. The documented reasons for late elective first-case start times are tabulated in Table 3. While no reason was documented in $23 \%$ of cases, the next most common reason was 'Surgeon not available' (15\%). After analysis of each delayed start, it was determined that $62 / 136(46 \%)$ were avoidable.

\section{Early terminations}

Early terminations accounted for 20100 minutes lost, $16 \%$ of total available time. The mean (SD) time available after the final case of the day for each theatre was 76 (70) minutes. Of the elective lists, 85/184 (46\%) were underrunning with $>90$ minutes of unused time after the last elective case was completed. This accumulated to a total of 12920 lost minutes or $10 \%$ of all available theatre time, which could have been occupied by an additional four minor cases ( $\sim 00$ minutes' duration) per day. The reasons for early terminations were poorly documented and are listed in Table 3. Early terminations of slates as a result of staff shortages (including nursing staff, surgeons and anaesthetists) accounted for 38\% of early terminations, while under-booked lists accounted for $13 \%$.

\section{Over-running time}

Over-running of lists occurred in 21/184 elective lists (11\%), totalling 1180 overtime minutes and representing just $0.9 \%$ of overtime over scheduled time. The majority of over-running minutes consisted of late finishes from the orthopaedic disciplines (1 069 minutes).

\section{Changeover time}

Total changeover time was 24300 minutes, representing $19 \%$ of all available time. The mean (SD) turnover time for the theatre complex was 24 (20) minutes. For elective theatres, mean (SD) turnover time was 20 (17) minutes. Mean (SD) turnover time for emergency theatres was 31 (30) minutes. For emergency theatres, changeover time accumulated to 11200 minutes (28\% of avail- able emergency theatre time). Prolonged turnovers, defined as $>60$ minutes between cases, accumulated to 8230 wasted minutes, representing $7 \%$ of total available time. Prolonged turnover occurred in 48/655 elective cases (7\%) and 56/359 emergency cases (16\%).

\section{Anaesthesia time}

Anaesthesia time was calculated to be 22920 minutes, $18.3 \%$ of total available time. The mean (SD) anaesthetic time per case was 23 (19) minutes.

\section{Surgical time}

Surgical time totalled 46260 minutes, 37\% of total available time. The mean (SD) surgical time was 46 (38) minutes.

\section{Intraoperative and slate delays}

There were 152 intraoperative and slate delays noted over the study period (Table 3). Delays in patient transport to the theatre complex occurred in $32 / 152$ (21\%) of delays. Only $5 / 152(3 \%)$ delays (3\%) were due to patients being inadequately prepared in the ward. Surgical or surgeon-related delays accounted for 50/152 delays (33\%), while delays due to anaesthesia and nursing occurred in 28/152 (18\%) and 23/152 (15\%), respectively. Just over half $(57 \%)$ of intraoperative and slate delays were avoidable.

\section{Theatre utilisation}

Overall operating theatre utilisation (theatre utilisation $=$ surgical time + anaesthesia time/ total available time) for the study period was $55 \%$. Marked differences existed in utilisation for the different disciplines (Table 2). Fig. 1 compares time utilisation per theatre with overall times. Table 2 displays the time utilisation of individual theatres and overall time utilisation of the theatre complex.

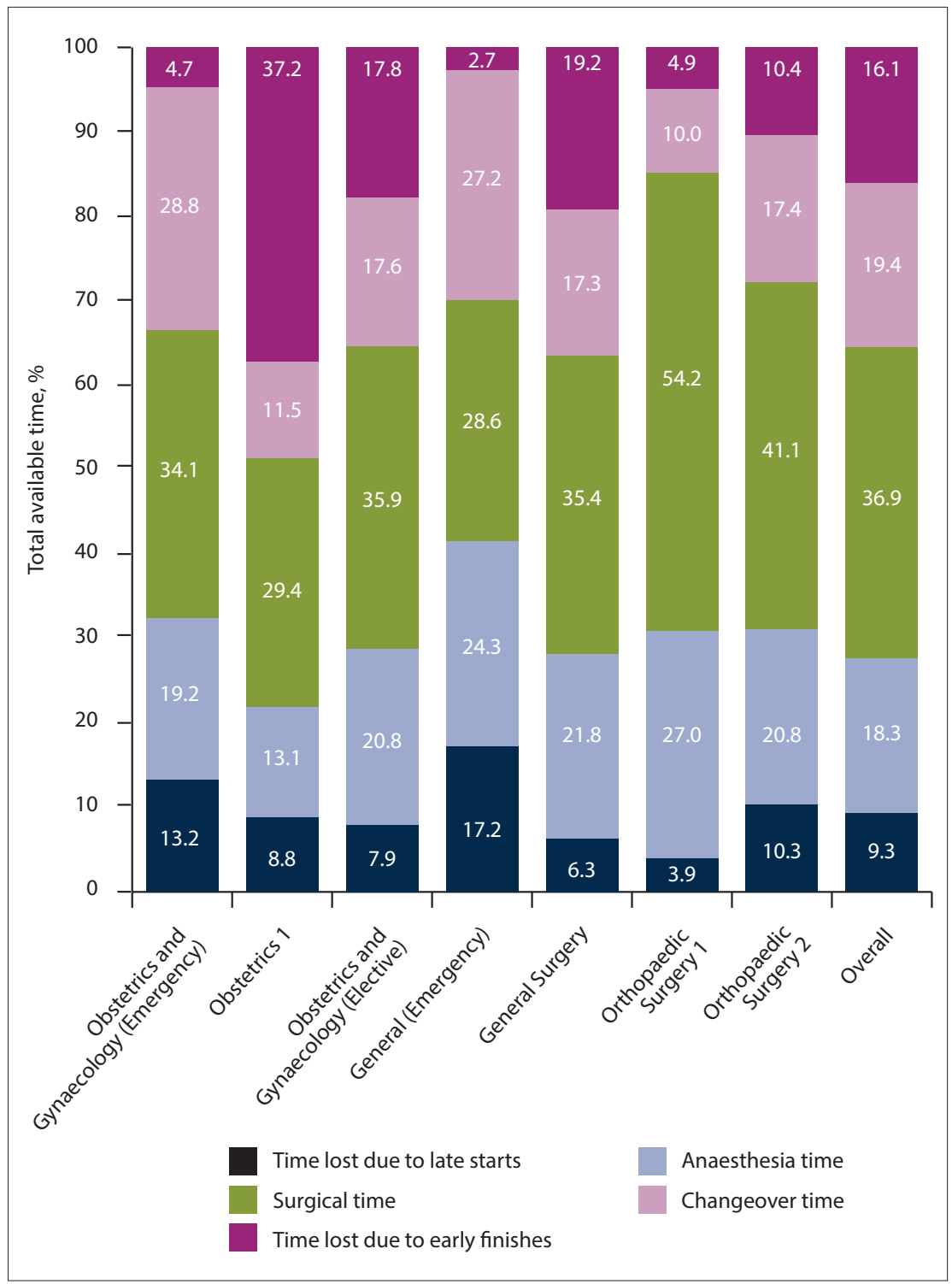

Fig. 1. Time utilisation by individual theatres compared with overall utilisation. 
Table 3. Reasons for slate time delays or early terminations and DOSCs

\begin{tabular}{|c|c|}
\hline & $n(\%)$ \\
\hline \multicolumn{2}{|l|}{ Reasons for late elective first-case starts $(N=136)$} \\
\hline Reason for delay not documented & $31(22.8)$ \\
\hline Surgeon not available & $21(15.4)$ \\
\hline Departmental meeting over-running & $19(14.0)$ \\
\hline Patient transport delayed & $17(12.5)$ \\
\hline Theatre not prepared & $14(10.3)$ \\
\hline First-case cancellation & $10(7.4)$ \\
\hline Nursing delay & $9(6.6)$ \\
\hline Anaesthetist not available & $9(6.6)$ \\
\hline Delayed patient preparation by ward & $3(2.2)$ \\
\hline $\begin{array}{l}\text { Elective case delayed due to emergency case } \\
\text { on table }\end{array}$ & $3(2.2)$ \\
\hline \multicolumn{2}{|l|}{ Reasons for intraoperative/slate delays $(N=152)$} \\
\hline Patient transport from ward delayed & $32(21.1)$ \\
\hline Prolonged/difficult surgical positioning & $19(12.5)$ \\
\hline Anaesthesia: technical delay & $19(12.5)$ \\
\hline Unanticipated difficult surgery & $15(9.9)$ \\
\hline Operating theatre not prepared & $15(9.9)$ \\
\hline Surgeon late & $12(7.9)$ \\
\hline Necessary equipment absent or malfunctioning & $12(7.9)$ \\
\hline Anaesthesia: patient-related complication & $9(5.9)$ \\
\hline Delayed transfer to recovery unit/ICU/mortuary & $5(3.3)$ \\
\hline Patient not prepared adequately by ward & $5(3.3)$ \\
\hline Order of surgical list changed & $4(2.6)$ \\
\hline Nursing delay due to lack of nursing staff & $3(2.0)$ \\
\hline Other & $2(1.3)$ \\
\hline \multicolumn{2}{|l|}{ Reasons for early elective list terminations $(N=85)$} \\
\hline Reason for early termination not documented & $39(45.9)$ \\
\hline Terminated due to staff shortages & $32(37.6)$ \\
\hline List under-booked & $11(12.9)$ \\
\hline Unplanned air-conditioning maintenance & $2(2.4)$ \\
\hline Patient not prepared for theatre & $1(1.2)$ \\
\hline \multicolumn{2}{|l|}{ Reasons for DOSCs $(N=232)$} \\
\hline Lack of available operative time & $94(40.5)$ \\
\hline $\begin{array}{l}\text { High temperature secondary to air-conditioner } \\
\text { failure }\end{array}$ & $36(15.5)$ \\
\hline Patient did not arrive for elective surgery & $29(12.5)$ \\
\hline Unknown reason & $17(7.3)$ \\
\hline Surgery no longer required & $13(5.6)$ \\
\hline Surgeon unavailable & $11(4.7)$ \\
\hline Replaced by emergency case & $8(3.4)$ \\
\hline Preop surgical or anaesthesia work-up needed & $6(2.6)$ \\
\hline Patient refused surgery & $6(2.6)$ \\
\hline Change in medical status of patient & $5(2.2)$ \\
\hline Patient not starved for theatre & $4(1.7)$ \\
\hline Equipment not available/broken & $3(1.3)$ \\
\hline
\end{tabular}

DOCSs = day-of-surgery cancellations; ICU = intensive care unit.

Orthopaedic theatres had the highest utilisation rates, $81 \%$ and $62 \%$, respectively. The Orthopaedic Surgery 1 theatre achieved the highest overall utilisation (81\%). It also had the highest actual operating time (54\%), while minimising wasted time from delayed starts (4\%) and early terminations (5\%), as well as the shortest changeover times, occupying just $10 \%$ of available time. The utilisation rate for the General Surgery elective theatre was 57\%. Utilisation in the two elective obstetrics and gynaecology theatres was $43 \%$ and $57 \%$, respectively. The Obstetrics 1 list's poor utilisation was partly due to shortages of surgeons and nursing staff causing delayed first-case starts and forcing early terminations of the list. A large proportion of unused time in the emergency theatres was due to unused times prior to first-case starts and prolonged changeover times, giving them utilisation rates of 53\% (Obstetrics and Gynaecology Emergency) and 53\% (General Emergency), respectively; nevertheless, these theatres had the highest output of total case numbers.

\section{Day-of-surgery cancellations}

In total, 232 elective cases were cancelled on the day of surgery, representing $26 \%$ of scheduled cases. The reasons for DOSCs are tabulated in Table 3. The commonest reason for cancellation was lack of available operative time (41\%). Surgeon unavailability was the reason for cancellation in 5\% of cases. Few patients were cancelled as a result of incomplete surgical or anaesthesia work-up $(3 \%)$ or a change in the medical condition of the patient requiring postponement of the surgery (2\%). On analysis of the reasons for cancellations, 112/232 (42\%) were avoidable, which could have led to a reduced cancellation rate of $14 \%$.

\section{Discussion}

The main findings of our study were that operating theatre utilisation was suboptimal when compared with the international benchmark of $70-80 \%{ }^{[6]} \mathrm{A}$ considerable amount of time appears to be lost each day as a result of late starts, prolonged turnovers and unexplained early terminations. This lost time contributed to the high DOSC rate in the study, which also falls above the $5 \%$ target of efficient theatres. ${ }^{[7]}$

The operating theatre utilisation of SA state operating theatres has been reported as $30-40 \%$, although this figure is not supported by published data ${ }^{[6]}$ A study from an SA private hospital group showed overall theatre utilisation of $48 \%{ }^{[6]}$ Similar international studies have shown varying utilisation rates of $50-90 \%,{ }^{[4,5,10]}$ depending on the site and size of the hospital. Optimising theatre utilisation and minimising DOSC is important when considering long waiting times for elective surgery in SA state hospitals, which range from months to several years. ${ }^{[1]}$ Patients waiting more than 6 months for surgery have been shown to experience increased anxiety, depression and unhappiness. ${ }^{[12]}$

Characteristics of an efficient theatre service include prompt starts, quick changeovers, minimal intraoperative delays and few overrunning cases. ${ }^{[13]}$ Efficiency must begin with appropriately booked elective operating lists. Inappropriate scheduling of elective cases, which set a theatre complex up for inefficiencies from the outset, has been highlighted in other studies. ${ }^{[14]}$ In our study, inappropriately booked lists based on random allocation were employed, leading to under- and over-booked lists that failed to take predicted surgical durations into consideration. While many other centres have the technology to schedule efficient theatre bookings based on scientific models, this is currently not feasible in our setting. ${ }^{[4]}$

Another important area requiring remedy is the delayed first-case start times seen in the study. It was noted that the complex lacks institutionally defined start times. Start-time delays and a lack of uniform definition of start times have also been shown in other studies. Start times are less useful than other measures of theatre efficiency, as studies have shown that late start times do not predict that a list will be inefficient or that it will over run. ${ }^{[14]}$ Macario $^{[7]}$ also demonstrated that a delay in start time of 45 minutes does not preclude efficient performance of the operating theatre list. Implementation of 
staggered start times may therefore be a consideration across the theatre complex in order to improve efficiency and minimise delays. However, it may impact on end times and shifts for doctors and nurses, which are often fixed in the state sector. Whether theatre managers should enforce strict start times can be debated; however, staff should still arrive on time and start times should still be well defined in order to interpret efficiency data. ${ }^{[14]}$

The present study found that a significant amount of time was wasted each day as a result of early terminations, and the underlying reasons for these need to be explored further. What also needs to be considered in the state sector is that early terminations do not necessarily mean early days for theatre users, as surgeons are often called to assist in areas of need out of theatre, and anaesthetists are required to perform pre-anaesthetic rounds in preparation for the next day. While appropriate booking and scheduling of lists may aid in minimising wasted time due to under-running lists, appropriate scrutiny of lists terminating early needs to be made by senior surgeons and anaesthetists.

Punitive methods, although not ideal, have been shown to improve theatre utilisation in some studies. ${ }^{[15]}$ Such measures may involve fining surgeons or removing theatre time from specialties with underbooked lists, ${ }^{[15]}$ and are unlikely to be useful in the SA state sector, which lacks financial incentives to drive caseloads. Designating more time to efficient disciplines, such as the Orthopaedic Surgery 1 theatre team in our study, may seem more reasonable. This theatre's utilisation exceeded $80 \%$ at the cost of increased over-running of cases, suggesting that it may be functioning above capacity and therefore may require additional theatre time. However, providing this may not be feasible, as shortages of staff in many disciplines, especially the orthopaedic discipline, mean that they are often unable to run more than one daily elective theatre list and cope with out-oftheatre responsibilities.

Prolonged changeover times occupied a significant proportion of available time in our study, as the theatre complex largely functions on linear processing of patients. Preparations for the next patient therefore only commence once the previous patient has left the theatre and the operating room has been fully prepared for the next case. While the reasons for this situation are multifactorial, lack of staffing is a significant contributor. It extends across all disciplines, including shortages of porters and cleaning staff, single-anaesthetist theatres and nursing staff shortages that delay theatre preparation and make the performance of parallel processing impossible in our complex. Parallel processing involves the anaesthetic team beginning the anaesthesia process in a preoperative holding area while the operating room is being turned over from the previous patient, ${ }^{[16]}$ and it has been put forward as a way to reduce changeover and room-anaesthesia times. While it would initially involve greater costs to the state, as pre-induction rooms would have to be set up and more anaesthetic and nursing staff employed, the excess costs are often buffered by improved theatre utilisation and case output. ${ }^{[4]}$ Parallel processing has been shown to be most effective in highturnover, short-case, full-day operating theatre lists rather than over all theatres. ${ }^{[16]}$

While the DOSC rate (26\%) in the present study was well above the $<5 \%$ target of efficient theatres, cancellation rates internationally (quoted by Bhuiyan et al. ${ }^{[17]}$ ) have been shown to vary between $5.6 \%$ and $25 \%$ depending on the site and size of the hospital. Locally, Bhuiyan et al. ${ }^{[17]}$ reported a DOSC rate of $44.5 \%$ in a Limpopo hospital, mainly as a result of elective theatres being required for emergency cases. In our study the low number $(3 \%)$ of cases that were cancelled because they were replaced by an emergency case highlighted the value of the two dedicated emergency operating theatres available in the complex. International studies, however, have questioned the efficiency of dedicated emergency theatres, as improved efficiency has been demonstrated when emergency theatres are closed and patients are instead operated on during reserved times on elective lists. ${ }^{[18]}$ In our setting, with a high surgical trauma burden, unpredictability and increased demand pose specific challenges, making the presence of available emergency theatres necessary in most centres. ${ }^{[2]}$ Nevertheless, many DOSCs could have been prevented by more effective communication, firstly between surgeons and patients to ensure that patients know when to present to hospital for surgery (this may be in the form of telephonic reminders to patients scheduled for elective surgeries), ${ }^{[4]}$ and secondly between surgeons and anaesthetic and nursing staff to ensure that preanaesthetic work-ups are completed and the equipment required is functional and available for the day of surgery.

Lack of available operative time was stated as the most common reason for DOSCs in the present study and appears to be due to the misuse of available time rather than an absolute lack of time. Time lost due to late starts, changeover times of $>60$ minutes and early terminations with $>90$ minutes remaining at the end of the list accumulated to 32790 minutes, representing $26 \%$ of total available time and $\sim 800$ lost minutes per day. It could be argued that the theatre could increase its capacity and perform an additional 360 cases of $~ 90$ minutes duration, which would have significantly increased utilisation and obliterated the DOSC rate. Conversely, it may be argued that the theatre complex is above capacity and that certain theatres could be shut down, redirecting staff to ensure efficient use of fewer theatres. These arguments are complex, as the duration of surgery is often unpredictable, unexpected complications may arise, and unanticipated resource constraints in SA state theatres are common. In addition, utilisation rates exceeding $90 \%$ are less than ideal, as they lead to over-running cases and increased delays. ${ }^{[19]}$ Theatre users must first demonstrate optimal use of currently available time before the decision is made to add or remove available time.

\section{Study strengths and weaknesses}

Staff were not blinded to the study, which may have affected their performance during the observation period, although this is unlikely. The completion of documentation relied on the anaesthetist, which may bias reporting towards the other specialties, although other disciplines were also encouraged to report any concerns to the recording anaesthetist. We were unable to evaluate the functioning of the post-anaesthetic care unit and the departure of the patient back to the ward. We were also not able to comment on out-oftheatre activities that impact on theatre efficiency, including patient transport and patient readiness for theatre, except when these resulted in a delay. An evaluation of adequate staffing was beyond the scope of this study, but directly affects theatre efficiency. Delays documented, such as those occurring during patient transport to the operating theatre, those due to surgeon and anaesthetist unavailability and nursing delays, may in fact be a result of inadequate staffing and over-stretched theatre personnel. Further studies evaluating the inefficient use of time due to staff shortages in state operating theatres are required.

\section{Conclusions}

Literature on state operating theatre efficiency in SA is lacking. Our measured theatre utilisation is higher than the previously quoted figures for SA state hospitals. However, a significant amount of time 
is wasted during the operating theatre day as a result of non-operative activities, contributing to a high on-day case cancellation rate. Before more theatre time can be made available, our theatre users must first optimise use of currently available time. Performance benchmarks for SA state operating theatres are required in order to set realistic goals that operating theatre managers can strive for.

\section{Practical recommendations}

- Standardised booking forms with time allocations per case and predicted operating times to aid in appropriate scheduling of cases.

- Clearly defined first-case start times that must be communicated to all theatre users. The feasibility of staggered start times can be explored by theatre managers.

- Senior anaesthetists and surgeons should scrutinise potentially early-terminating lists, and plans for adding an additional case to the list should be made early.

- A minor surgical case should be pre-emptively dedicated as the first emergency theatre case of the day, to allow prioritising of the subsequent emergency cases while minimising wasted time from delayed starts.

- Scheduling of a single high-output list monthly may serve to limit long surgical waiting times. Additional staff should be reallocated to this list to aid with parallel processing of patients for the list.

- Institutionally based performance benchmarks to be set in order to measure efficiency and track progress.

- Regular monitoring of operating theatre efficiency data. Our modified measurement tool (available online at http://www.samj. org.za/public/sup/asmal_13815.pdf) can be used as a template for theatre managers to collect data on a regular basis, in order to measure performance and track progress. Completion of the template is simple and cost-effective.

- Plans for the introduction of electronic recording systems should be made, as these will make data readily available, allowing for easy monitoring of theatre performance.

Declaration. This publication was submitted as a requirement for completion of IIA’s MMed (Anaesth) degree.
Acknowledgements. None.

Author contributions. All authors contributed equally to the writing, data analysis and proofreading of the article.

Funding. None.

Conflicts of interest. None.

1. Archer T, Macario A. The drive for operating room efficiency will increase quality of patient care. Curr Opin Anesthesiol 2006;19(2):171-176. https://doi.org/10.1097/01.aco.0000192796.02797.82

2. Van As A, Brey Z, Numanoglu A. Improving operating theatre efficiency in South Africa. S Afr Med J 2011;101(7):444-448. https://doi.org/10.1111/ans.12013

3. Verguet S, Alkire BC, Bickler SW, et al. Timing and cost of scaling up surgical services in low-income and middle-income countries from 2012 to 2030: A modelling study. Lancet Glob Health 2015;3(Suppl 2):S28-S37 https://doi.org/10.1016/S2214-109X(15)70086-0

4. Keller A, Ashrafi A, Ali A. Causes of elective surgery cancellation and theatre throughput efficiency in an Australian urology unit. F1000Res 2014;3:197. https://doi.org/10.12688/f1000research.4824.1 5pyet C. An audit of the use of ophthalmic theatre time. Community Eye Health 2002;15(44):61-62.

6. Hartmann D, Sunjka B. Private theatre utilisation in South Africa: A case study. S Afr Med J 2013;103(5):285-287. https://doi:10.7196/SAMJ.6460

7. Macario A. Are your operating rooms 'efficient?' Anesthesiology 2006;105(2):237-240. https://doi. org/10.1097/00000542-200608000-00004

8. Faiz O, Tekkis P, Mcguire A, Papagrigoriadis S, Rennie J, Leather A Is theatre utilization a valid performance indicator for NHS operating theatres? BMC Health Serv Res 2008;8(1):28. https://doi. org/10.1186/1472-6963-8-28

9. Lankoandé M, Bonkoungoua P, Kib BK, et al. Economic and psychological burden of scheduled surgery cancellation in a sub-Saharan country (Burkina Faso). South Afr J Anaesth Analg 2017;23(6):145-151. https://doi.org/10.1080/22201181.2017.1379788

10. Vinukondaiah K, Ananthakrishnan N, Ravishankar M. Audit of operation theatre utilization in general surgery. Natl Med J India 2000;13(3):118-120.

11. Dunn RN, Kavalieratos T, Nortje M. Hip and knee arthroplasty waiting list - how accurate and fair? S Afr Med J 2017;107(4):323-326. https://doi.org/10.7196/SAMJ.2017.v107i4.12145

12. Lizaur-Utrilla A, Martinez-Mendez D, Miralles-Muñoz FA, Marco-Gomez L, Lopez-Prats FA. Negative impact of waiting time for primary total knee arthroplasty on satisfaction and patientreported outcome. Int Orthop 2016;40(11):2303-2307. https://doi.org/10.1007/s00264-016-3209-0

13. Testi A, Tanfani E, Torre G. A three-phase approach for operating theatre schedules. Health Care Manag Sci 2007;10(2):163-172. https://doi.org/10.1007/s10729-007-9011-1

14. Pandit J, Abbott T, Pandit M, Kapila A, Abraham R. Is 'starting on time' useful (or useless) as a surrogate measure for 'surgical theatre efficiency'? Anaesthesia 2012;67(8):823-832. https://doi. org/10.1111/j.1365-2044.2012.07160.x

15. Wright JG, Roche A, Khoury AE. Improving on-time surgical starts in an operating room. Can J Surg 2010;53(3):167-170.

16. Friedman DM, Sokal SM, Chang Y, Berger, DL. Increasing operating room efficiency through parallel processing. Ann Surg 2006;243(1):10-14. https://doi.org/10.1097/01.sla.0000193600.97748.b1

17. Bhuiyan M, Mavhungu R, Machowski A. Provision of an emergency theatre in tertiary hospitals is . Bhuiyan M, Mavhungu R, Machowski A. Provision of an emergency theatre in tertiary hospitals is
cost-effective: Audit and cost of cancelled planned elective general surgical operations at Pietersburg cost-effective: Audit and cost of cancelled planned elective general surgical operations at Pietersburg
Hospital, Limpopo Province, South Africa. S Afr Med J 2017;107(3):239-242. https://doi.org/10.7196/ Hospital, Limpopo Province, South Africa. S Afr Med J 2017;107(3):239-242. https://doi.org/10.7196/
SAMI.2017 v107i3. 10687

18. Wullink G, van Houdenhoven M, Hans EW, van Oostrum JM, van der Lans M, Kazemier G. Closing emergency operating rooms improves efficiency. J Med Syst 2007;31(6):543-546. https://doi. org/10.1007/s10916-007-9096-6

19. Tyler DC, Pasquariello CA, Chen CH. Determining optimum operating room utilization. Anesth Analg 2003;96(4):1114-1121. https://doi.org/10.1213/01.ane.0000050561.41552.a6

Accepted 29 March 2019 